\section{OFFICERS, 1967}

President: Dr. Oswald Savage.

President-elect: Dr. J. J. R. Duthie.

Hon. Treasurer: Dr. Dudley Hart.

Senior Hon. Sec.: Dr. J. A. Cosh, Royal National Hospital for Rheumatic Diseases, Bath.

Junior Hon. Sec.: Dr. A. J. Popert.

Hon. Librarian: Dr. W. S. C. Copeman.

Clinical Meeting held at the White Hart Hospital, Harrogate, on May 20 to 21, 1966.

DR. T. G. REAH (Harrogate) opened the proceedings by giving a short paper concerning a family where grandfather, father, and son all suffered from rheumatoid arthritis.

Discussion.-DR. J. S. Lawrence (Manchester): A family of three generations of males all suffering from rheumatoid arthritis is clearly unlikely to occur by chance. It provides, moreover, convincing evidence that sex linkage on the X-chromosome is not involved in any genetic cause which may be present.

DR. REAH presented the case of a woman with a destructive lesion of the bones, particularly affecting the hands. Biopsy of a gland showed it to be a reticulum cell sarcoma.

Discussion.--DR. J. V. WILSON (Harrogate): I should think that the age of the patient was of interest here, as well as the distribution of the disease. Was the serum uric acid estimated?

DR. REAH: The patient attended another hospital 6 months ago, when the serum uric acid was found to be $7.3 \mathrm{mg} . / 100 \mathrm{ml}$. She was given colchicine and the level fell to $5 \mathrm{mg}$. $/ 100 \mathrm{ml}$. While she has been here, the serum uric acid has been around $5 \mathrm{mg} . / 100 \mathrm{ml}$. The diagnosis at the other hospital was said to be "atypical gout".

Pleural Lesions and Pulmonary Infections in Rheumatoid Arthritis, by DR. W. C. WALKER (Leeds).: 516 patients with classical or definite rheumatoid arthritis were studied clinically, radiographically, and serologically and were compared from the respiratory point of view with 301 patients with degenerative joint disease. The incidence of pleurisy, pleural fibrosis, and pleural effusion was determined and measures in the diagnosis of rheumatoid pleuritis assessed. Features of the arthritis, other systemic complications, results of serological and

\section{PROGRAMME OF MEETINGS, 1967}

March 17: Royal National Orthopaedic Hospital, Stan more.

June 17: Heberden Round conducted by Dr. Barbara Ansell, Canadian Red Cross Memorial Hospital, $\mathbb{\complement}$ Taplow.

November 17/18: Annual General Meeting at the Well- $\overrightarrow{0}$ come Foundation, Euston Road, London.

November 17: Heberden Oration by Dr. L. E. Glynn.

November 17: Annual Dinner at the Royal College of Physicians, Regents Park, London.

haematological tests, and radiographic changes in those with rheumatoid pleural effusions were compared with the remainder of the rheumatoid population. The incidenceof respiratory infections in the two groups was compared and their significance in rheumatoid arthritis discussed.

Discussion.-DR. J. V. WILsON (Harrogate): Has any $\frac{3}{0}$ body found RA cells in pleural effusions?

DR. WALKER: We have looked for them only once andthey were not present.

Prof. J. H. Kellgren (Manchester): Do these patiegts come from urban or rural areas?

DR. WALKER: They came mostly from the polluted atmosphere of Leeds; some came from urban areas around Leeds and a few from rural areas.

Prof. E. G. L. Bywaters (Taplow): This excellen paper contains much material for discussion. When Aronoff, Fearnley, and I* investigated lung lesions ir rheumatoid arthritis, we used a control series both in the $x$-ray series of 130 rheumatoid patients and in the post mortem series of 42 rheumatoid patients, and in both there was a relative preponderance of lung lesions in the rheumatoid series, involving bronchitis, pulmonary fibrosis, and pleural thickening in the radiological series and bronchitis, pleural adhesions, and bronchiectasis irb the autopsied series. We concluded, however, that there was no specific lung lesion common enough to appear in this small post-mortem series. The controls were choserg on a random basis for both series.

DR. J. S. LAWRENCE (Manchester): I have recently hadP occasion to review patients with positive sheep cell tests and a control group with negative tests. They had chest $x$ rays and then a forced expiratory volume test. A highe proportion of positive sheep cell tests was found in thoser with a low expiratory volume. The chest $x$ rays did show more pleural thickening in the sero-positive group. In this, as in most population samples, few of the seron positive people had evidence of rheumatoid arthritisct Nevertheless, we found that pleural thickening was mor£ common in those with arthritis than without. Of course positive sheep cell tests are more common in urbarf populations, so we cannot be sure to what extent this ha\& interfered with the conclusions. It does rather support ${ }^{+}$ Dr. Walker's contention that these pulmonary infections 
might be more causative than the result of rheumatoid arthritis.

*Brit. med. J. (1955). 2, 228.

Green Serum in Rheumatoid Arthritis, by DR. K. D. SHAH and DR. V. WRIGHT (Leeds): Certain patients with rheumatoid arthritis were found to have green serum. This could be seen, but not measured spectrophotometrically. The colour moved with albumin, and did not appear to be due to free verdoperoxidase. It was photosensitive and was not reproduced by dilution of serum or bilirubin. It appeared to be due to a relative absence of yellow pigment. The phenomenon was correlated with the clinical, serological and radiological manifestations of the disease.

Discussion.-Prof. E. G. L. Bywaters (Taplow): You say it is easy to recognize: presumably all these readings were done blind?

Dr. Wright: It is easier to see the green when the serum is frozen. The readings were done by three observers independently without knowledge of the diagnosis; the definite yellows and definite greens were always put into the right category, but the intermediate group had a definite observer error in calling them either intermediate or yellow.

Prof. E. G. L. Bywaters (Taplow): How did you categorize them?

DR. WRIGHT: We took the definite green group and compared them with the rest.

DR. M. JefFrey (Harrogate): I wonder if Dr. Shah tried to correlate this finding with the serum iron. We know that serum iron may be low in active and chronic rheumatoid disease. I wonder if the green colour is simply due to a lack of the normal pink of siderophyllin.

DR. SHAH: We could not do this investigation on many patients because of technical difficulties, but Mr. Clough, our biochemist, demonstrated in some the point you make. However, iron is bound to the globulin fraction not the albumin.

Investigation of Morning Stiffness, by DR. T. G. PlunketT (Leeds): As an extension of the investigation of morning stiffness of rheumatoid arthritis, a clinical and physiological study was undertaken. A self-recording pneumatic dynamometer was designed and a portable arthrograph constructed. An inter-relationship of diurnal variation of strength of grip and physical stiffness was suggested. Evidence was presented to show that weakness of grip is insufficient of itself to account for the phenomenon of morning stiffness. The effects of sleep (natural and induced), immobilization, exercise, and temperature were studied.

Discussion.-Dr. O. SAVAge (London): You measure your strength of grip in lb. per sq. in. Most of us test grip in $\mathrm{mm}$. Hg. Could you tell us what are the equivalent scales?

DR. V. Wright (Leeds): The average rheumatoid patient would grip in the region of $5 \mathrm{lb}$. per sq. in. compared with $200 \mathrm{~mm}$. Hg.

DR. D. A. BREWERTON (London): There are two points about the measurement of grip that have always bothered me. One is that muscles and joints were mentioned, but not the tendons in between. The other is the habit of talking about strength as if it were a single entity. It all depends on how you measure strength. For instance, when measuring a static muscle contraction in the presence of a painful joint, it makes a considerable difference whether the muscle is attempting to shorten against a fixed resistance or whether it is holding an external force.

DR. J. T. ScotT (London): It is difficult to relate morning stiffness to other factors. A few years ago I studied morning stiffness in a few patients with rheumatoid arthritis, estimating passive stiffness in a metacarpophalangeal joint with an extension spring.* Objective joint stiffness was greater in the morning than later in the day. Strength of grip was also weaker in the early morning, and hand volume, measured by water displacement, was greater. However, a similar variation in hand volume and strength of grip, but not in joint stiffness, was found in normal subjects.

Dr. Plunkett: I am in fact looking into the effect of immobilization and oedema on strength of grip. I had a little difficulty in measuring hand volume, but I have now devised a method of doing it more accurately than by water displacement. I am using an alginate gel that the Dental Department use for making impressions of the mouth. I take a cast of the hand, which sets in about 1 to 2 minutes, and measure the volume of this.

Dr. V. Wright (Leeds): May I make a point on the question of tendons. It is obvious that tendons do contribute to stiffness at certain phases. Working with the cat wrist joint, which is comparable in stiffness to the metacarpophalangeal joint of a child, we found that in the normal physiological range of joint motion tendons contribute little to overall stiffness. Their effect is a check-rein action at the extremes of joint movement. OP the point of static muscle contraction, I entirely agree that the two modes Dr. Brewerton has described will yield different results, but in the case of strength of grip the method Dr. Plunkett has used seems to be the nearest to the physiological situation.

Dr. D. A. Brewerton (London): But cats don't have rheumatoid arthritis!

DR. V. WRIGHT (Leeds): That is correct.

*Scott, J. T. (1960). Ann. rheum. Dis., 19, 361.

Antibody Response in Patients with Rheumatoid Arthritis, by Dr. R. B. PaYne, DR. J. W. Czenalowski, and DR. V. WRIGHT (Leeds): There is evidence to suggest that an aetiological factor in the production of rheumatoid arthritis might be a deranged antibody response of such patients. To test this hypothesis the antibody response to particulate and soluble antigens was tested over a period of 52 days in a group of patients with rheumatoid arthritis and in a control group with osteoarthrosis, and the results were reported. Preliminary results showed that in a patient with Felty's syndrome there was no immunological response to the injection of antigen. The other results at this stage were inconclusive.

Study of Lumbar Disk Degeneration, by Mr. A. NAYLOR (Bradford): The natural history of intervertebral disk herniation and degeneration shows that the symptomatology is characteristically phasic. The development of our hypothesis of disk prolapse and the biochemical and biophysical changes in disk degeneration were outlined, and possible auto-immune factors discussed.

Results of our biochemical and biophysical investiga- 
tions, which showed effects of changes on elasticity of disk and its mechanical efficiency, were reviewed. More recent investigations particularly concerned variations in non-collagenous proteins. Enzyme breakdown of polysaccharide/collagen complex was briefly outlined and its possible clinical significance discussed.

Discussion.-DR. V. Wright (Leeds): First, has Mr. Naylor found that in the lumbar spine biochemical changes occur in the disks at each level as well as the one which is prolapsed? Secondly, if there is an age distribution of the biochemical changes, does he find that a relatively young subject with disk protrusion does in fact have changes in the disks? How does he explain protrusions at a younger age?

Mr. NAYLOR: We take the protruded nucleus of these patients and part of the annulus fibrosis and examine the specimens biochemically. These show changes you expect in a much older patient. I only remove the prolapsed portion. We note that herniations in animals often occur at various levels of the spine. These herniations are usually seen in animals with disks which have aged prematurely. Certain breeds of dogs, such as the pekinese and dachshund, are very prone to disk degeneration; these breeds show intervertebral disk degeneration at about 3 or 4 months of life, though relatively resistant breeds do not show these changes. The biochemical changes I have described may well be the result of the process, not the cause.

Rheumatoid Peripheral Neuropathy, by DR. T. G. PlunketT and DR. J. R. Golding (Harrogate): A series of 64 patients with rheumatoid peripheral neuropathy was presented ( 28 males and 36 females), of whom 28 were dead, 26 alive, and ten untraced. Most had been diagnosed in the years $1959-60$, very few being seen after then. The onset of peripheral neuropathy in males was at an average age of 54, and in females at an average age of 51. The average survival time in the fatal cases after the diagnosis of neuropathy was 1 year in males and 2 years in females. The average age of the males was 63 and of the females 61 at the time of follow-up. The relationship to corticosteroid therapy and stress was discussed. Fatal cases and the post-mortem results were also reported.

Discussion.-DR. J. S. LAWRENCE (Manchester): I was interested in the histogram of the change in incidence. Have you looked into the relationship to gold treatment?

DR. GoldING: As far as we can tell more gold is being used in this region than 5 years ago, although the incidence of cases of rheumatoid peripheral neuropathy has diminished over the years. Not much was used in 1959-60 when most of the patients were alive. However, two French authors consider that widespread severe peripheral neuropathy may appear immediately after gold injections.

DR. V. L. STeINBERg (London): My findings in a similar number of cases confirm this trend in incidence in 195960-61, and in the last year I have only had one or two patients. All my findings are similar to those of Dr. Golding and $I$ agree that gold is not used in these patients more than in others. Were we using large amounts of new steroids in the 1960s?

$D_{R}$. Golding: We were probably using larger doses in 1960 than we are using now.
Prof. E. G. L. Bywaters (Taplow): You commented that arteritis was not present at autopsy in some cases. What type was their neuritis?

DR. GoldING: Widespread.

Prof. E. G. L. Bywaters (Taplow): I asked because it is sometimes very difficult to find evidence of arteritis. One particular case we had with asthma, severe eosino- $\overrightarrow{0}$ philia, and peripheral neuritis, died finally following retroperitoneal fibrosis, and routine study of sections $\overline{\bar{n}}$ failed to find any arteritis. With repeated blocks and $\frac{\bar{\sigma}}{\partial}$ many sections one or two examples of healed arteritis $\overparen{Q}$ could be shown.

DR. D. A. Pitkeathley (Manchester): I should like to ask first whether the three patients with leg ulcers had $\vec{\circ}$ punched-out lesions? and secondly what is being done with regard to corticosteroids when neuropathy develops? $\vec{\omega}$

DR. GoldING: My feeling is that once neuropathy develops, whatever you do about the dose of steroids, it is going to take its own course.

DR. J. T. ScotT (London): I do not believe that peri-c pheral neuropathy in rheumatoid arthritis necessarilyo carries the bad prognosis attributed to it by this study. It was not clear from the facts given that the patients who died always succumbed from their rheumatoid disease, 0 rather than from an intercurrent but unrelated cause. It is also possible that Dr. Golding's well-known interest inthis complication has led to the referral of very sick $Z$ patients.

I recently reviewed our experience of this condition with Dr. Pallis, ${ }^{*}$ and we found that prognosis could be related to the clinical pattern of neuropathy. For example, a purely sensory neuropathy carries a variable but on the whole not unfavourable outlook. A wide-s spread sensorimotor neuropathy, on the other hand, is almost always followed by death.

DR. GoldiNG: We have been on the look out for mindo cases and have not really found many of them. I men-s tioned that we were able to follow up only five patientso who had not been on steroids. They all have a simple form of neuropathy and are all still alive. I think theD prognosis is better in the non-steroid than in the steroid? treated cases.

DR. G. Holden (Worthing): In patients on steroids, was there any relationship to any particular steroid, or to changes from one steroid to another?

DR. GoldING: There was no relationship to any particular steroid.

DR. V. WRIGHT (Leeds): Do you have any informatioro on the period between the onset of symptoms and the times of diagnosis? The explanation of your histogram show ing decreasing incidence may be that, knowing youe interest, physicians have now referred their cases to you? and you have scooped the pool. Secondly, what type of neuropathy was it in the ten cases not followed up?

DR. Golding: As far as we can tell most cases wer seen soon after the development of symptoms. RegardI. ing the patients not followed up, their type of neuropathy was very evenly spread.

*Pallis, C. A., and Scott, J. T. (1965). Brit. med. J., 1, 1141.

Reduced Incidence of Osteo-arthritis in Weight-bearing Joints after Anterior Poliomyelitis, by MR. G. F. WALKER and DR. J. H. GLYN (London): The legs of 98 patients over 35 years old, who had suffered an attack of anterior poliog myelitis at least 10 years previously and in whom one leg was more severely paralysed than the other, were exam-

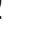


ined. The hip and knee radiographs of 100 unselected people, of the same sex and age group, taken during the Leigh field survey were also assessed. It was thus possible to make three comparisons: between radiographs of joints of weaker and stronger legs, and between each of these and those of a random group of control joints. Antero-posterior radiographs of knees and hips of the polio patients and control radiographs were read in random order by two of three observers. Considerable difficulty in fitting some of the polio joint radiographs into the classification of Kellgren and Lawrence was experienced, but this was overcome by assessing six parameters for each joint.

Osteo-arthritis occurred less frequently in weightbearing joints controlled by muscles weakened by poliomyelitis (polio joints), probably because of the effects of poliomyelitis rather than of a decrease in weight-bearing activity. Only one example of primary osteo-arthritis in a severely paralysed joint was found, but a few polio joints had developed osteo-arthritis after an intra-articular fracture. Weak muscles from poliomyelitis seemed to have a protective role, relative rather than absolute. It was not possible to prove that calipers protect polio joints. There was no increase in osteo-arthritis with obesity or with increase of weight-bearing activity. No close association of symptoms with radiological osteoarthritis was found; muscles or their attachments were thought to play an important role in producing joint symptoms.

It was striking that completely flail knees often retained ligamentous stability, and that neither stable nor unstable joints, if devoid of all muscle control, developed osteoarthritis.

Suggestions that patients with poliomyelitis do not develop osteo-arthritis because their activities are restricted or that joints of weaker limbs are spared by greater use of the stronger limbs were not substantiated.

Discussion.-DR. D. R. L. NeWTON (Middlesbrough): Is it quite correct that we should refer to "instability" of the hip as measured by Trendelberg's sign? Instability of the joints really infers a ligamentous laxity which would be most unlikely to occur in the hip joint.

DR. GLYN: This seems to me to be a point of semantics rather than of substance. What surprised me from the beginning was that patients who were suffering from this muscular imbalance, and who were consequently putting gross stresses on their joints, did not develop recurrent "sprained joints" with peri-articular pains. Furthermore, I should have expected such repeated traumata to lead eventually to degenerative arthritis.

DR. D. R. L. NewTon (Middlesbrough): Having regard to the anatomy of the hip, I think this would create a realignment of the weight-bearing area involved.

Prof. E. G. L. Bywaters (Taplow): How do you assess osteo-arthritis? I do not quite see why Dr. Glyn felt there was no evidence from his figures that lack of weightbearing was responsible for the relative immunity of the paralysed side.

DR. GLYN: Taking the second question first. The degree of weight-bearing did not vary in the different groups. The incidence is identical in patients who are walking and even doing a normal day's work and in those who are partially chair-bound. The arthritic changes therefore seemed to bear no relationship to the degree of $\stackrel{\mathbb{D}}{=}$ weight-bearing activity, but they did bear a relationship 3 to the degree of paralysis. With regard to the assessment $\square$. of osteo-arthritis, we used both the conventional clinical criteria and the six radiological parameters mentioned $\Rightarrow$ earlier. If two or more of these radiological parameters were positive, we diagnosed osteo-arthritis.

DR. V. WRIGHT (Leeds): What are the vascular changes in poliomyelitis? It could be that this is the major factor $\bar{c}$ in producing the changes of osteo-arthrosis and certainly in producing symptoms. There is a good deal of evi- $\varrho$ dence, particularly from phlebography before and after $\mathrm{g}$ intertrochanteric osteotomy, that alteration of vasculature ${ }^{\infty}$ is responsible for some symptoms in osteo-arthrosis. $\vec{\circ}$

DR. D. A. BREWERTON (London): There certainly are profound changes in the vasculature in poliomyelitis and $\vec{\omega}$ this may be bound up with changes in sympathetic $\mathcal{S}$ function.

DR. GLYN: We feel that the explanation of our clinical observations may possibly lie in these vascular changes.

Rheumatoid Arthritis after Poliomyelitis, by DR. E. N. $\dot{\sigma}$ GLICK (London): Thompson and Bywaters (1962) des- $\bigcirc$ cribed unilateral rheumatoid arthritis in the non-paralysed $\mathrm{O}$ limbs after hemiplegia. They noted that paralysis of both upper and lower motor neurone types have been reported $\vec{z}$ as protecting against degenerative arthritis, but they hado no information regarding the effect of lower motor $₫$ neurone paralysis on rheumatoid arthritis.

In five patients who developed rheumatoid arthritis $\mathbb{\Phi}$ after having had poliomyelitis, arthritis changes were $-\vec{T}$ more marked in non-paralysed limbs. The combinatiog of rheumatoid arthritis and polio may be rarer than might be expected from the prevalence of these to conditions.

Discussion.-Prof. E. G. L. Bywaters (Taplow): I should like to congratulate you on the paper. I was wondering if perhaps Dr. Lawrence might have any $\frac{\mathcal{D}}{2}$ figures on the separate incidences of these two diseases in $\varrho$ the population, and whether perhaps Dr. Glyn might $\overrightarrow{\vec{P}}$ comment on the incidence of rheumatoid arthritis in the $\frac{3}{3}$ polio population. From his 98 cases there ought to be something like one or two cases.

DR. J. GLYN (London): There was one, but I was unable to follow him up.

DR. J. S. LAWRENCE (Manchester): One out of 98 is? rather a low prevalence. The patients were over 35 윽 years of age.

DR. GLICK: I actually tried to contact this man, but did $\delta$ not get any reply. He was over 80 years old. As regards the incidence figures of polio, $I$ tried to find out $O$ what proportion of the population between the ages of $\frac{}{5}$ 20 and 60 had paralytic polio, but there are no figures. $D$

Dr. W. Watson Buchanan (Glasgow): Dr. Glick's observations perhaps lend support to Dr. Duthie's view N that rest, and in particular proper splintage, has an or important place in the treatment of rheumatoid arthritis. N

Evaluation of the Relative Roles of Hereditary and $\mathrm{N}$ Environmental Factors on Autoantibody Production, by Drs. W. W. Buchanan, J. A. Boyle, W. R. Greig, M. BARR, R. MCANDREW, R. B. Goudie, and J. R. ANDERSON (Glasgow): A study of 145 healthy twin pairs $\stackrel{\oplus}{+}$ was undertaken in an attempt to define the role of 0 heredity and environment in the production of autoanti- 
bodies in normal people. Five autoantibodies were studied, including rheumatoid factor by the Hyland agglutination technique and antinuclear factor using rat liver nuclei as antigen.

Comparison of autoantibody concordance rates in mono- and dizygous twins showed no evidence of significantly raised concordance in the monozygotic twins for any of the autoantibodies studied. These results suggested that in normal persons heredity is much less important than environment in autoantibody production.

Discussion.-DR. J. S. LAWRENCE (Manchester): This is a fascinating study. Certainly Dr. Ball did find aggregation of the sheep cell factor in the populations of Leigh and Wensleydale. When we came to divide the relatives into parents, siblings, and offspring, we found that most of the aggregation was occurring in the offspring. The siblings had a slight increase, and the parents had no more than the expected rate. This in turn did not seem to fit any genetic hypothesis. Recently Professor Collard of Manchester has been looking at the Salmonella and Brucella antibodies in persons with positive sheep cell tests in Leigh and Wensleydale, and he found some increase of antibodies in the sheep cell positive persons, suggesting that it is bacteria rather than genes which are handed down in these families.

Flufenamic Acid (CI-440) in the Treatment of Rheumatoid Arthritis, by DR. K. T. RAJAN (Stoke Mandeville): Flufenamic acid is a stable weak carboxylic acid, rapidly acting, anti-inflammatory, and analgesic compound with the chemical name of $\mathrm{N}$-(a,a,a-trifluro- $m$-tolyl) anthranilic acid.

A double-blind double-cross-over trial compared the relative analgesic properties in rheumatoid arthritis of flufenamic acid, aspirin, and phenylbutazone. Thirty patients were admitted to the trial; complete records were obtained from 26 patients and a partially completed record from one.

Discussion.-DR. J. T. ScotT (London): One hesitates to criticise such a carefully conducted trial, but do you not think it might have been a useful control to include a period of placebo tablets in your design?

DR. RAJAN: We did not feel justified in putting these patients who were suffering from active rheumatoid arthritis on inert tablets for 2 weeks.

DR. N. CARDoE (Norwich): We have just completed a similar trial in which we compared flufenamic acid and aspirin. We doubled the dose to 600 instead of $300 \mathrm{mg}$. At the end of the trial we did give an opportunity for these patients to continue on the drug, and we have now two patients who have had it for 18 months. They are both satisfied that they are doing well, but radiologically they have got worse. Three of the patients on this dose had to stop it because of diarrhoea.

DR. A. G. S. Hill (Stoke Mandeville): It seems to me that, because no inert drugs were used, the result is open to the two alternative interpretations, that none of the drugs had any analgesic potency, or that all were of similar potency. This trial was designed to answer the question, does flufenamic acid have greater analgesic power than aspirin or phenylbutazone.

DR. J. T. ScotT (London): Yes, but it is conceivable that an advantage of one analgesic over the other might not be demonstrated because the techniques were not sufficiently sensitive. You would suspect this if you found no advantage of either drug over the inert tablets.

DR. T. M. Chalmers (Edinburgh): The daily dose of aspirin used in the trial was slightly smaller than commonly used in routine treatment.

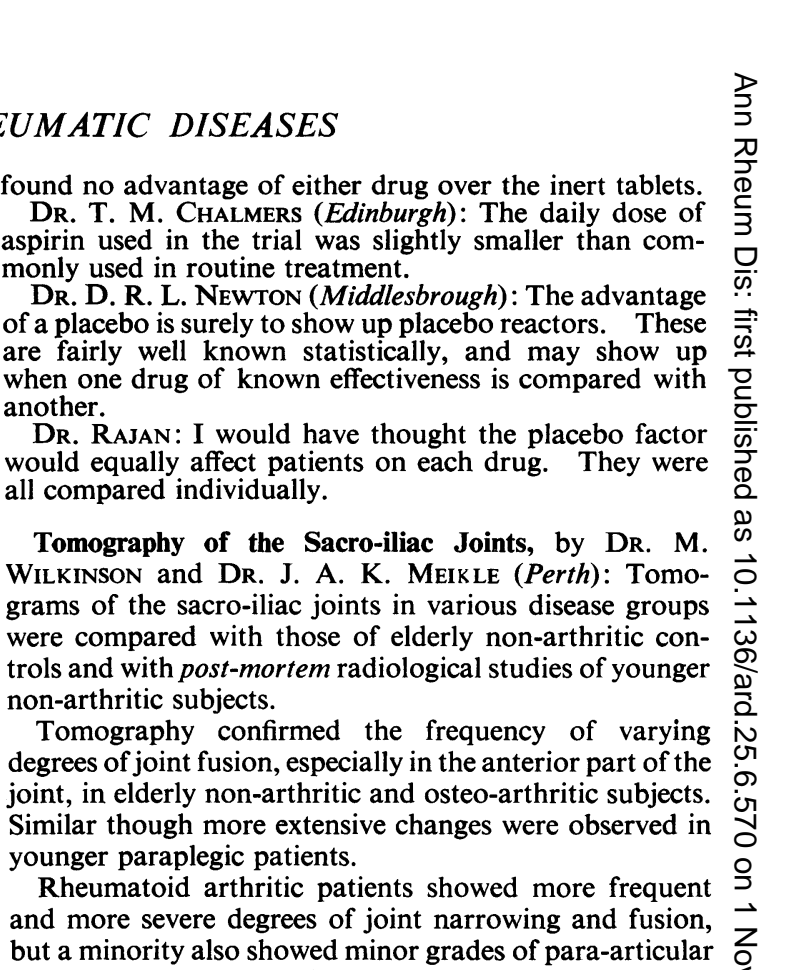

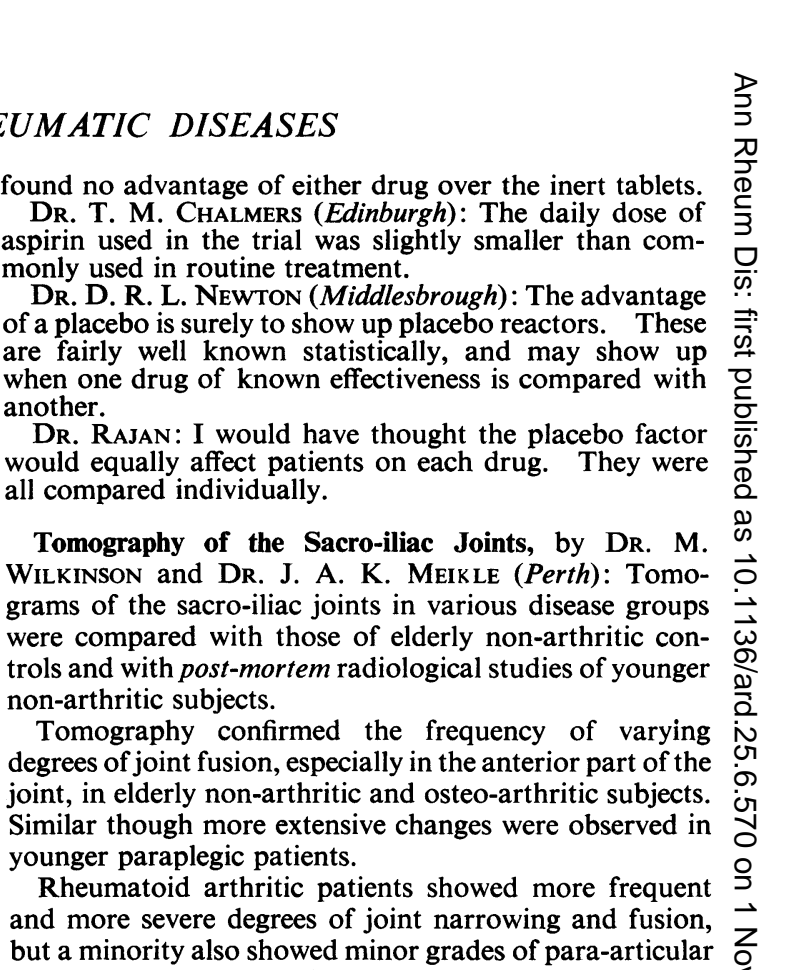

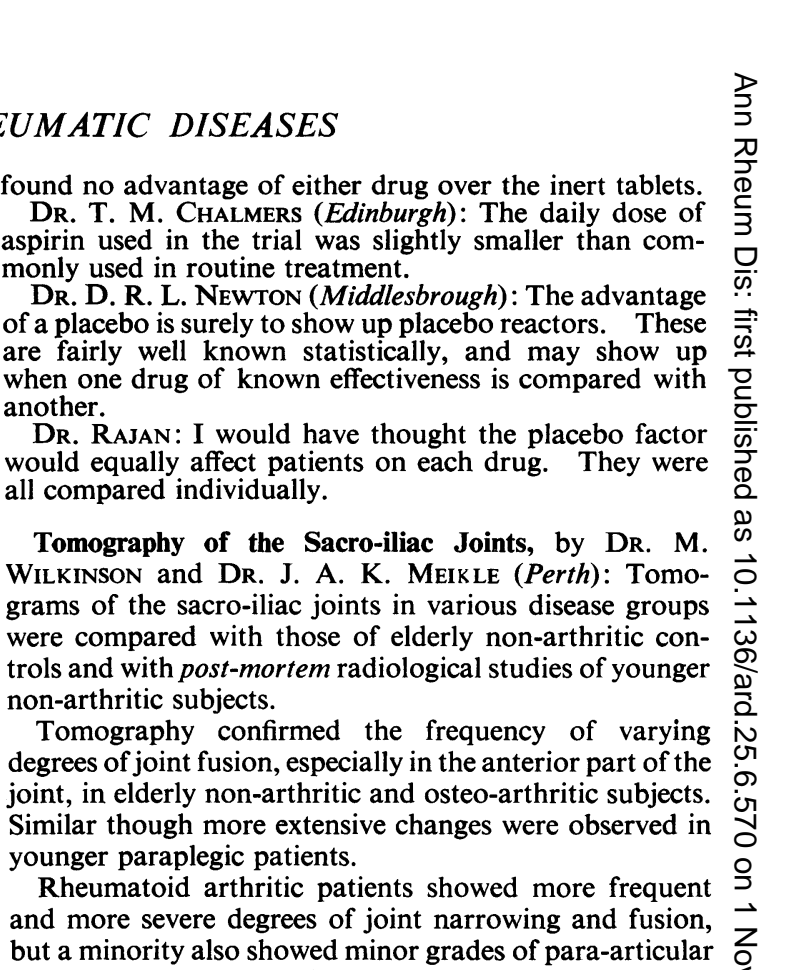

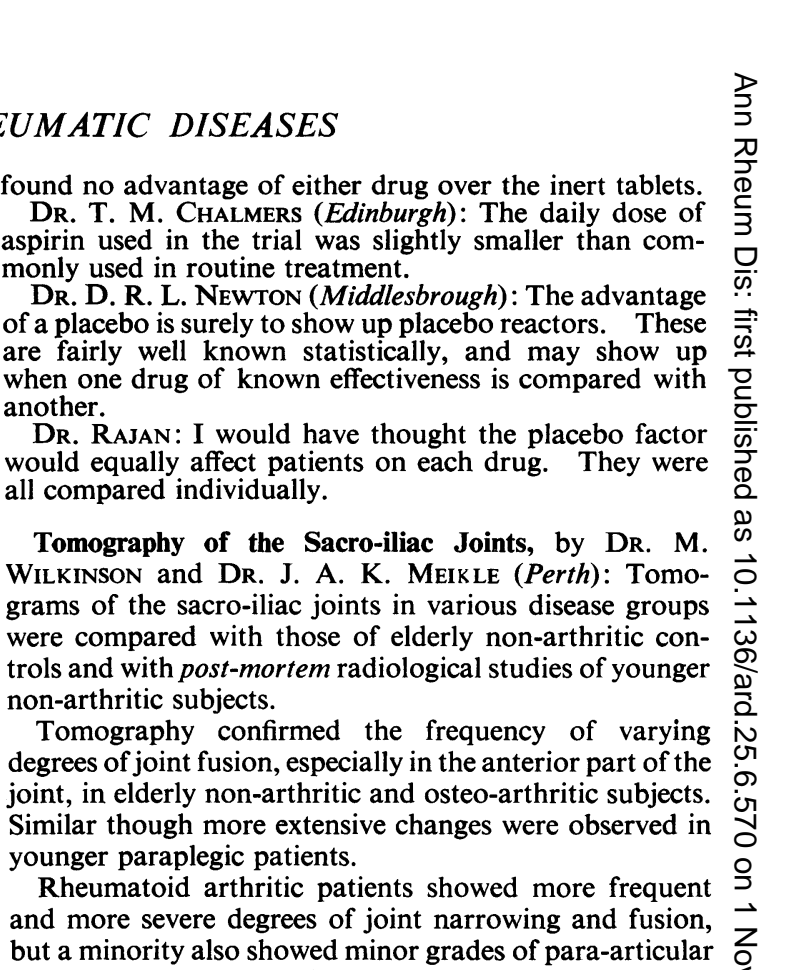
another.

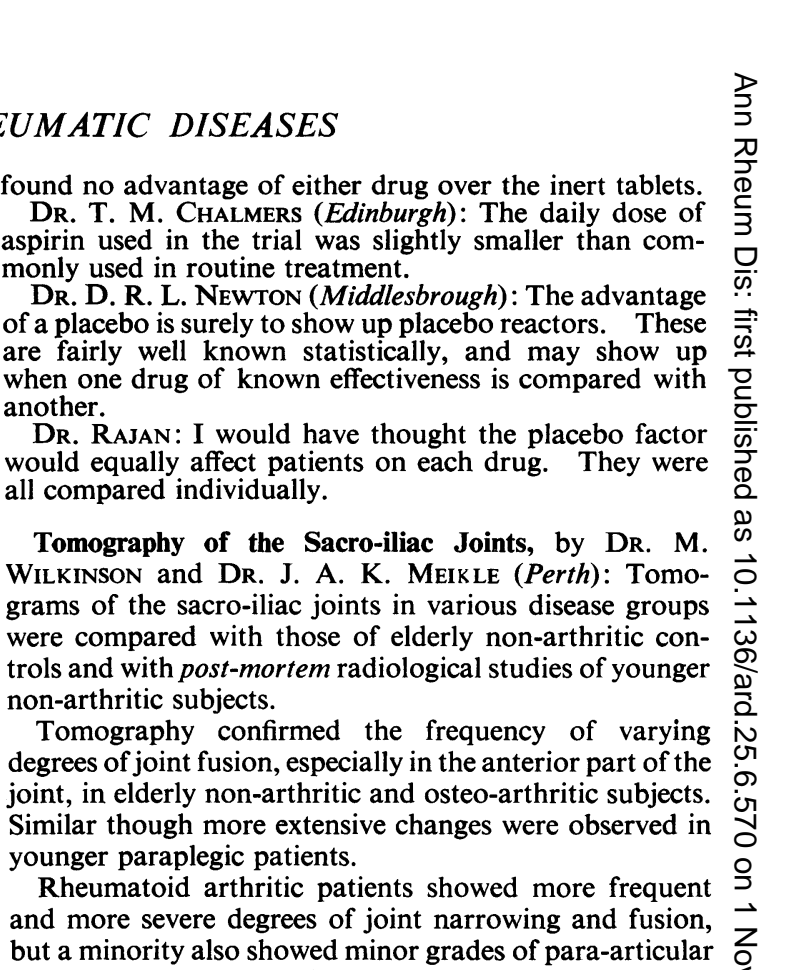

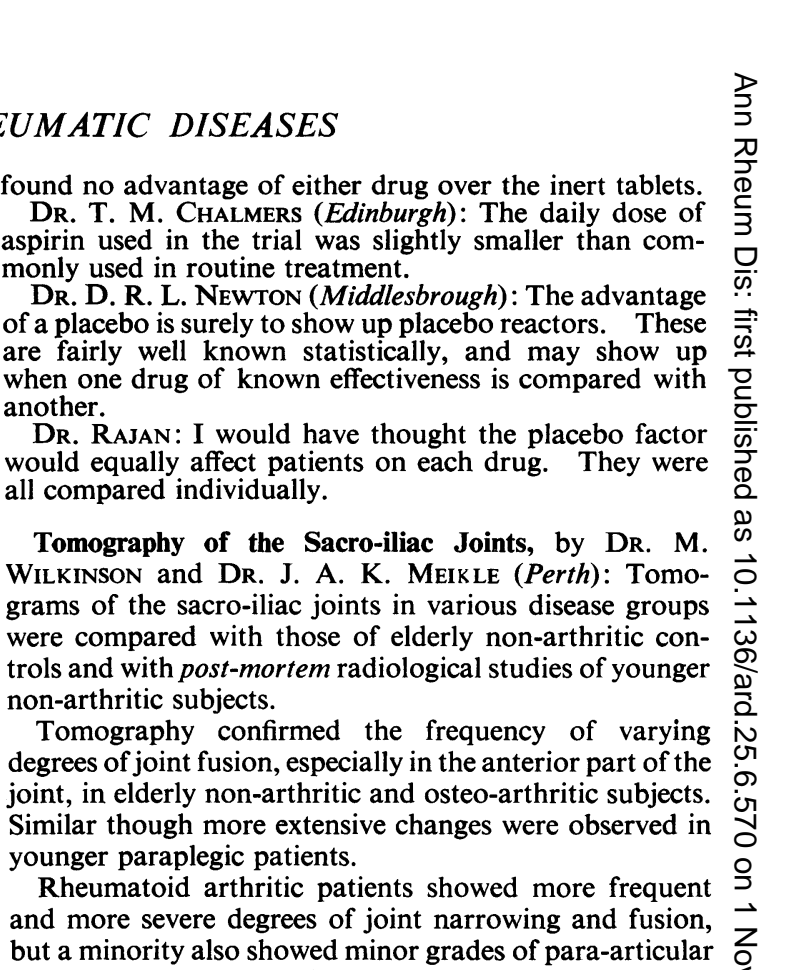

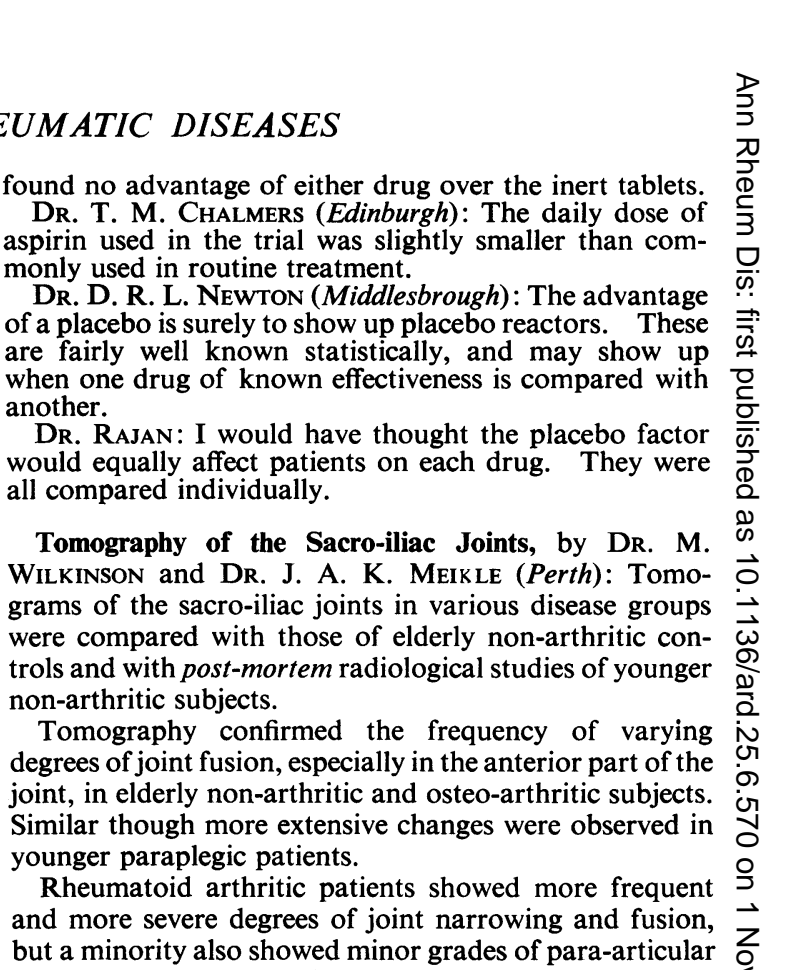

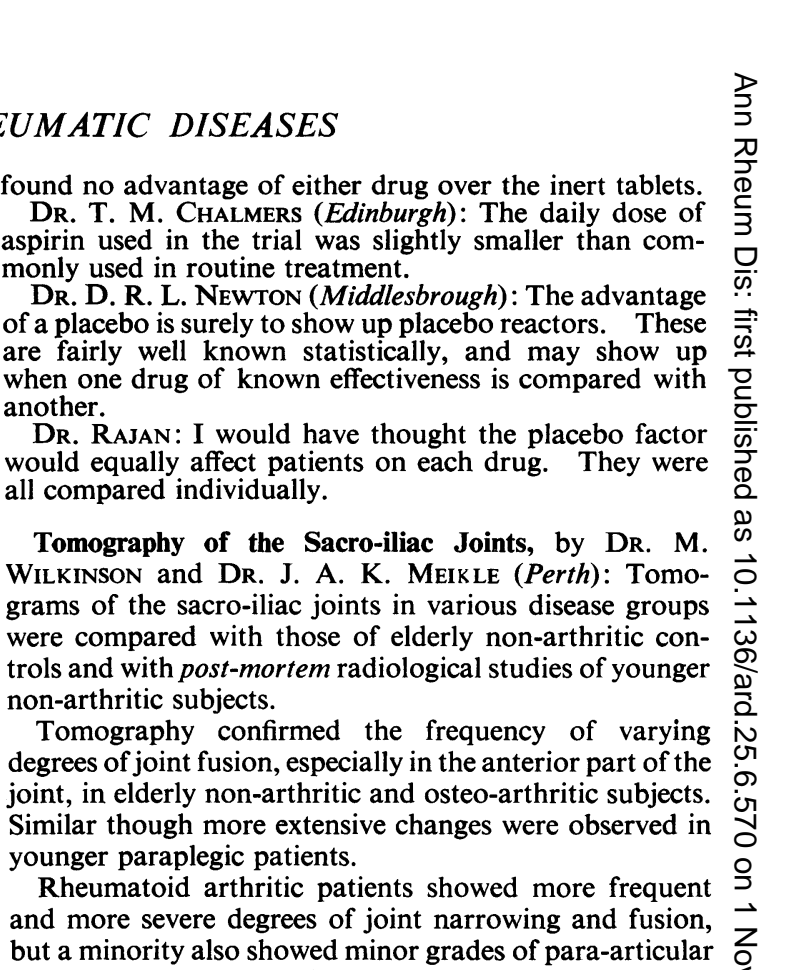

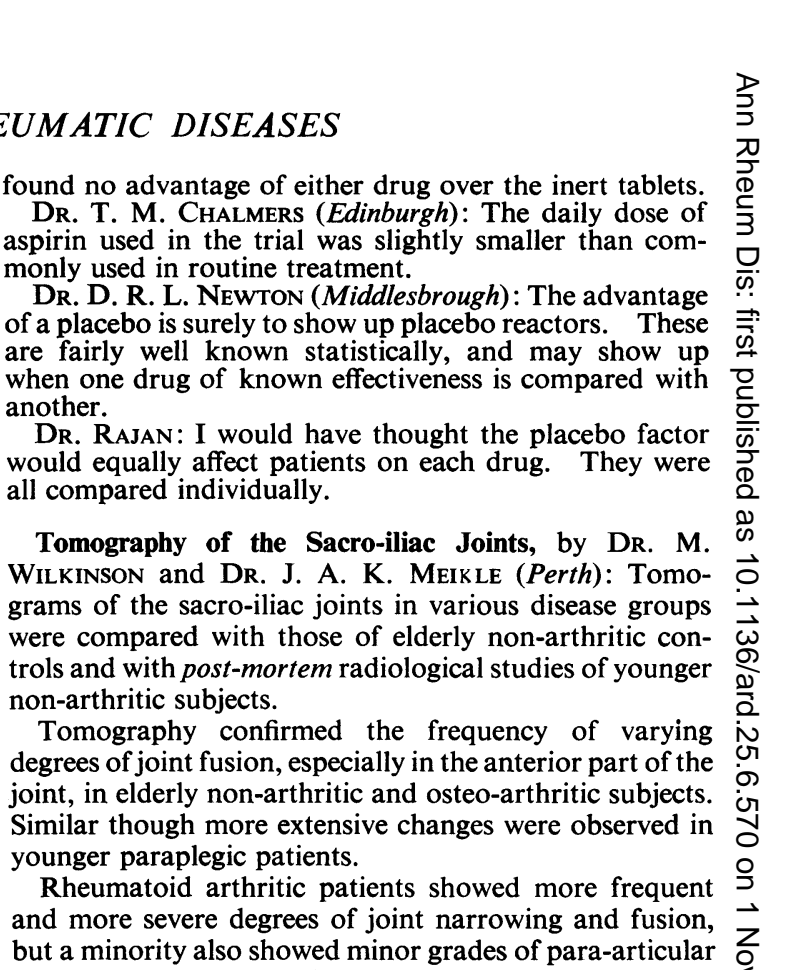

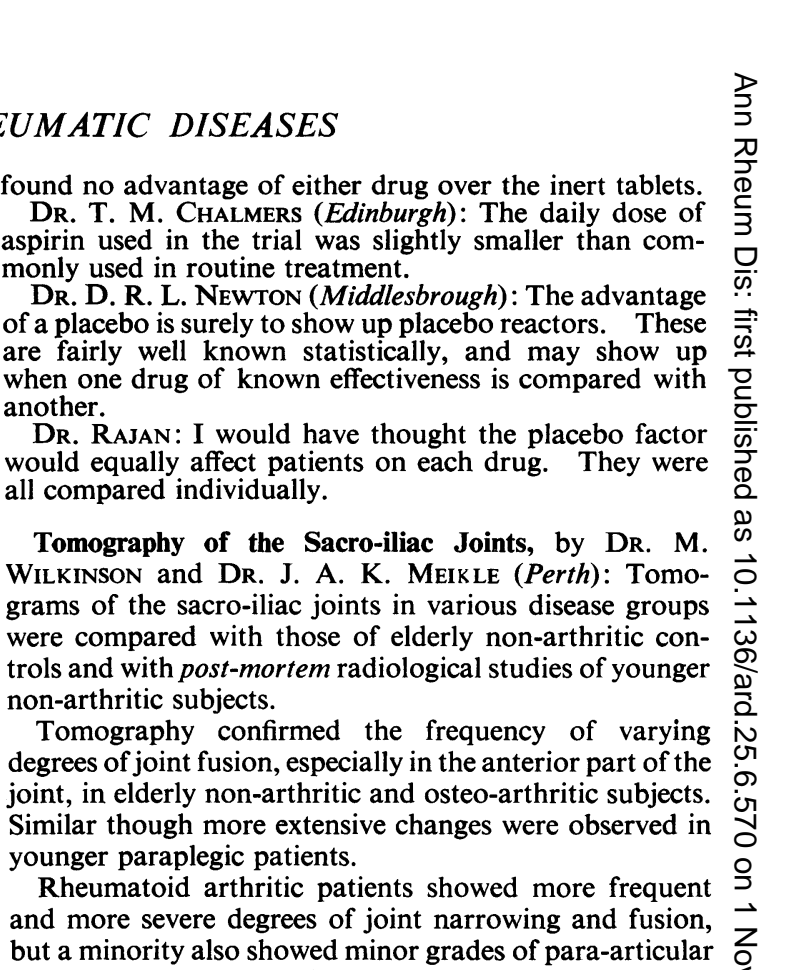

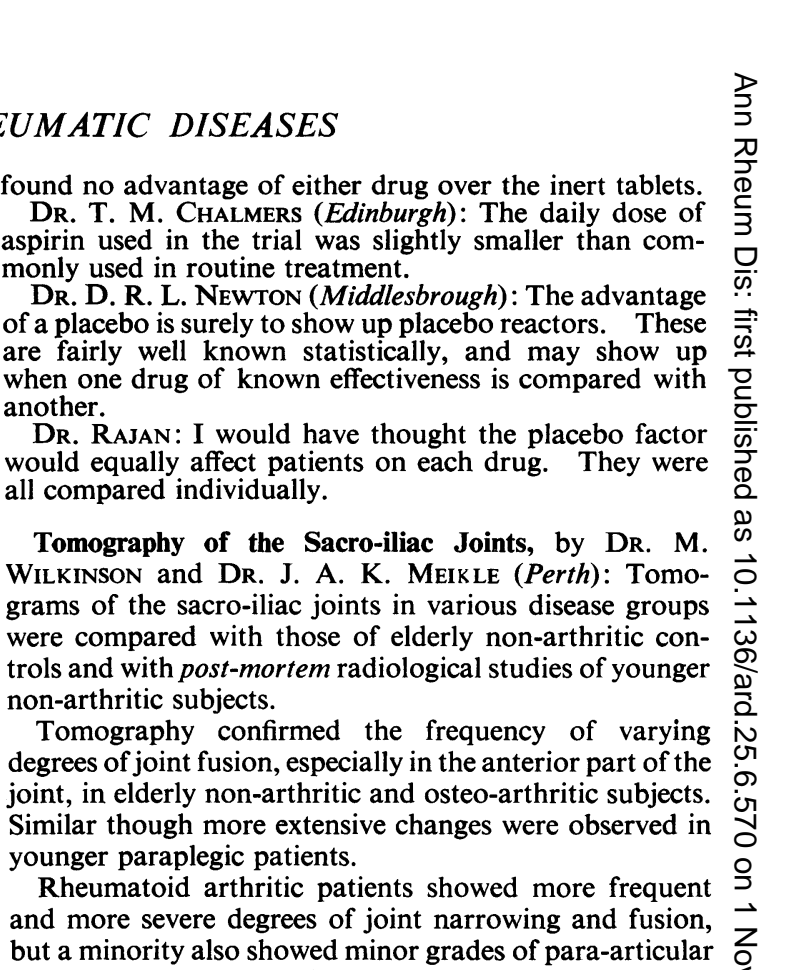

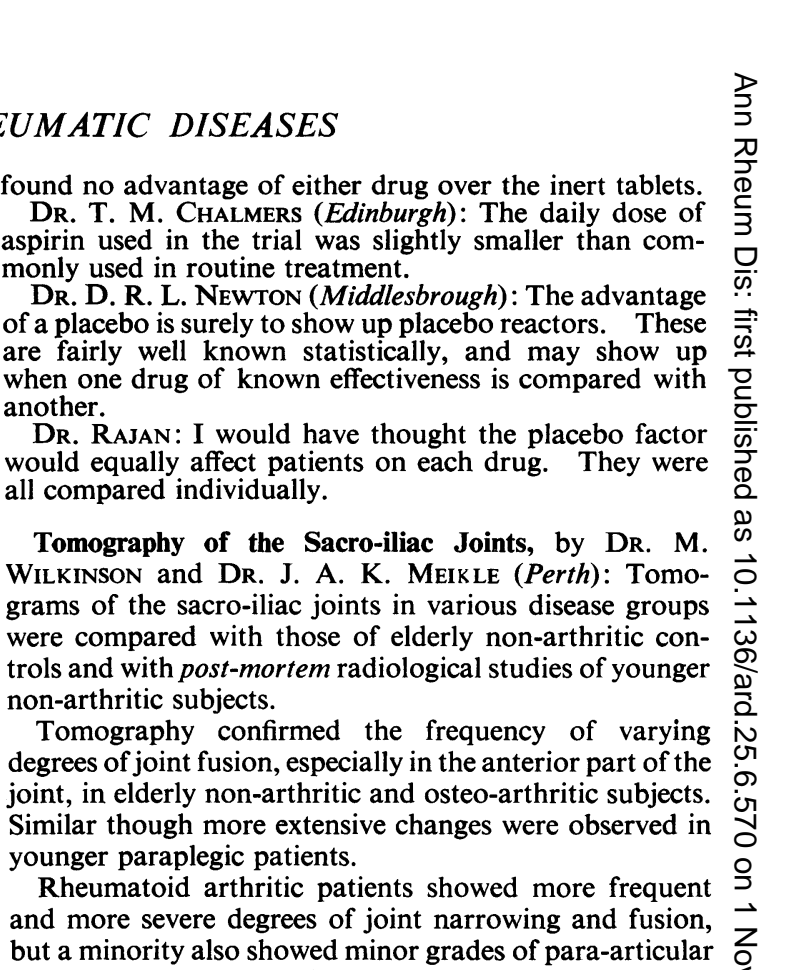
non-arthritic subjects.

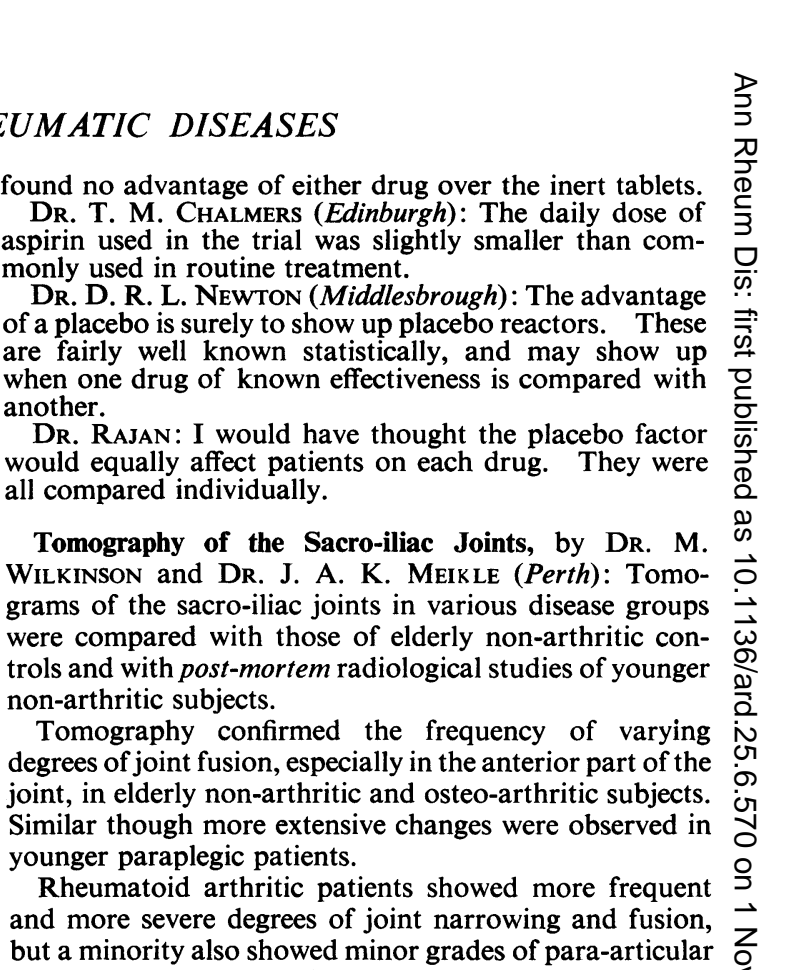

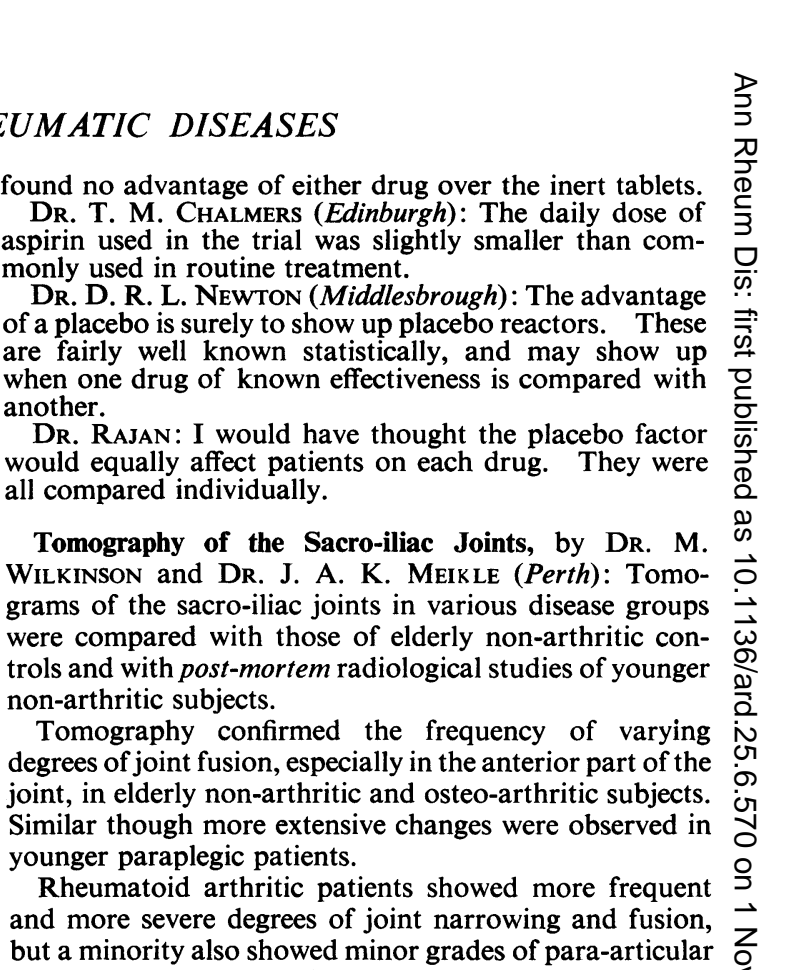

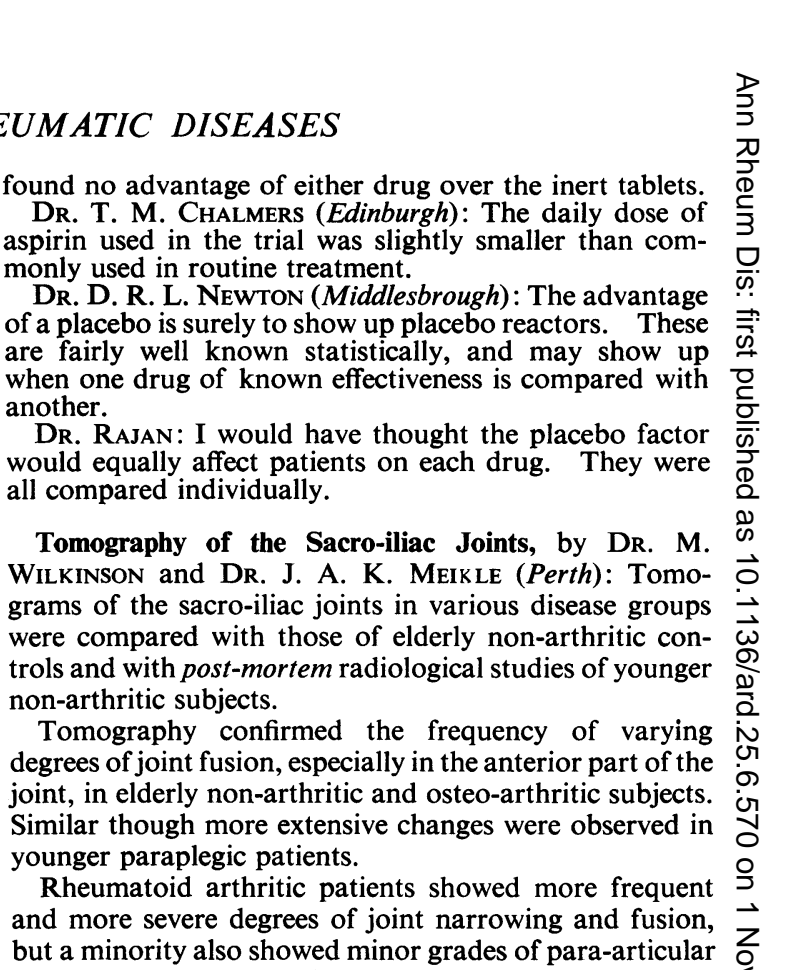

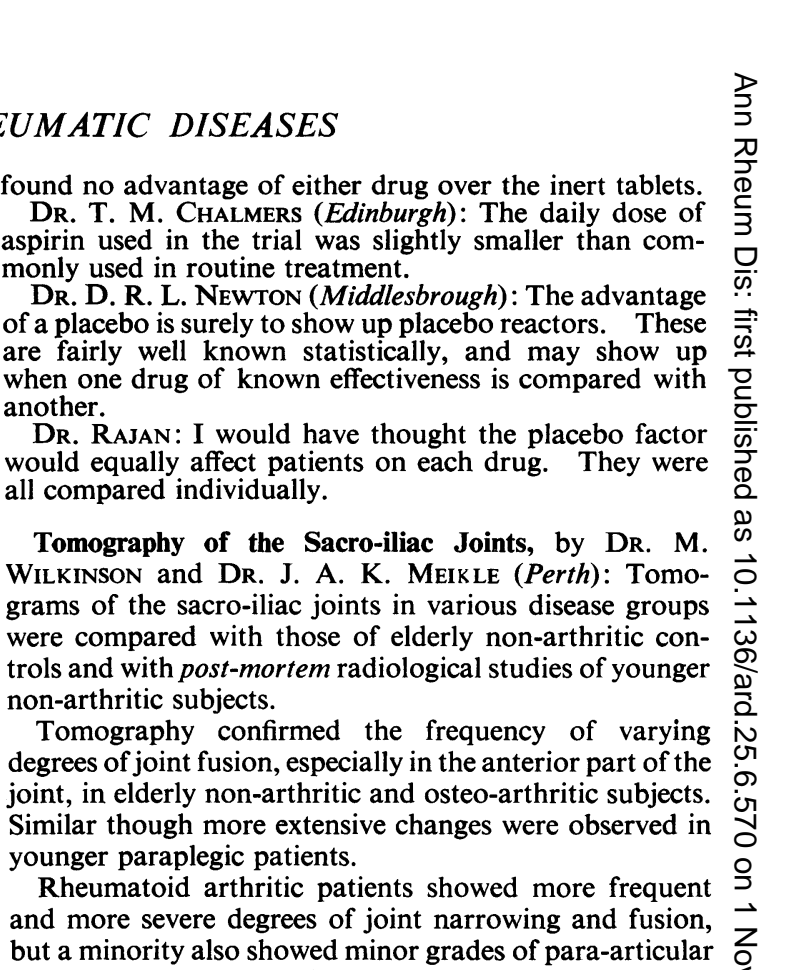
younger paraplegic patients.

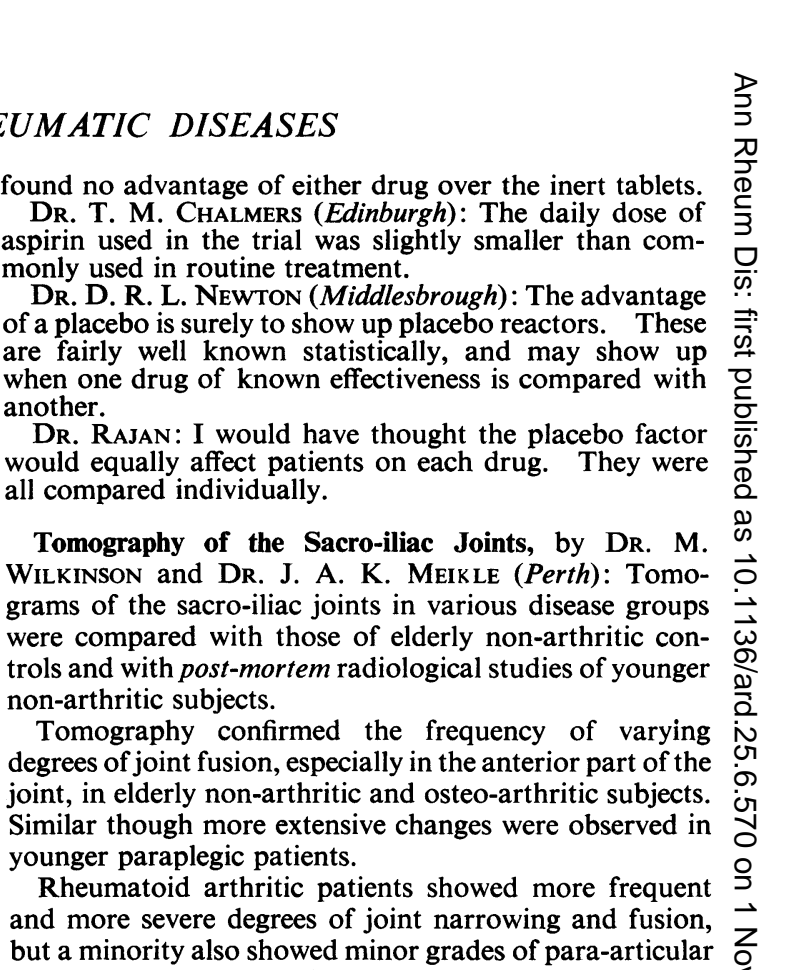

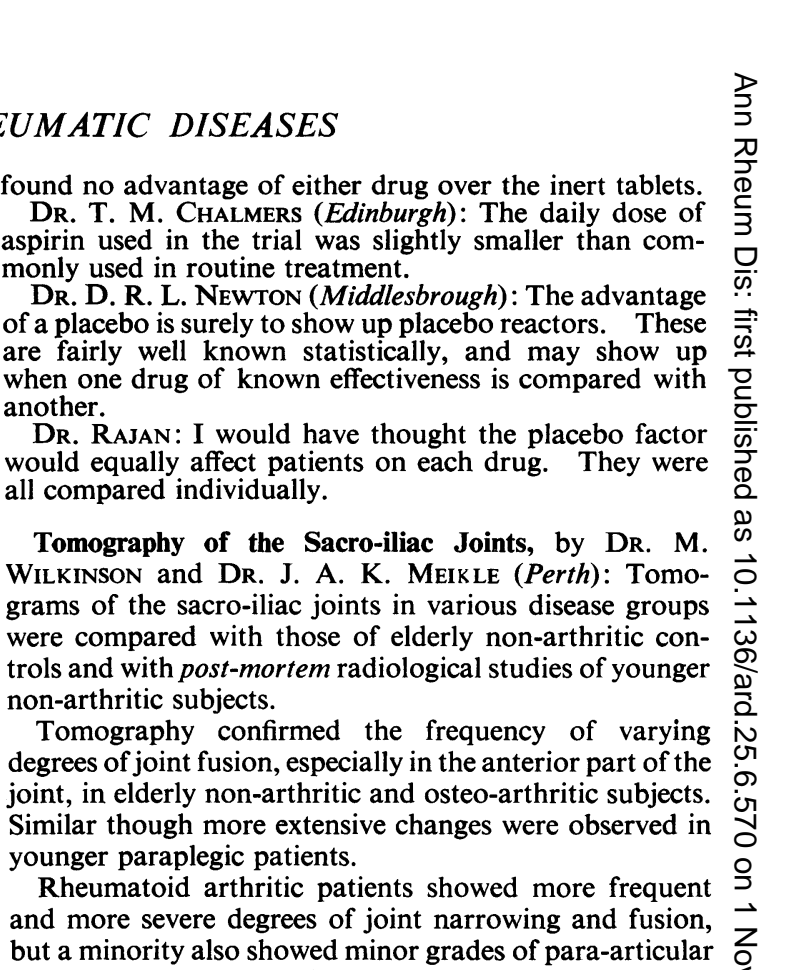
but a minority also showed minor grades of para-articular sclerosis and small erosions. In ankylosing spondylitis marked degrees of sacro-iliac joint narrowing and fusion were found at an early age, but of more use in diagnosis was the demonstration of para-articular sclerosis, marginal erosions, and occasionally pseudo-widening of the joint space, all of which tended to be more prominen than in rheumatoid arthritis.

Compared with antero-posterior radiographs of the sacro-iliac joints, tomograms were more useful for demonstrating joint narrowing and fusion but only slightly better for sclerosis, erosions, and osteophytes.

Discussion.-DR. V. WRIGHT (Leeds): I think this is an excellent piece of work, and am interested to see that Dr. Wilkinson did not find the changes of ankylosing spondylitis in the paraplegic patients. As you know, we presented before this learned Society evidence that the sacroiliac changes in these patients differed from the sacroiliitis of ankylosing spondylitis with which they had previously been confused. It is reassuring to have Dr. Wilkinson's confirmation.

DR. D. R. L. NEWTON (Middlesbrough): Some 10 years ago I was very dissatisfied with antero-posterior views, and I agree that oblique views are not generally helpful. Have we any information about comparison of tomograms with postero-anterior views?

DR. WiLkINSON: We do not have any information on this. We felt it was not justified to expose the patients to further radiation. I agree it would be useful for comparison.

DR. M. Thompson (Newcastle-upon-Tyne): I should not of wish to see the complete rejection of oblique views of the $N$ sacro-iliac joints. I have found them useful particularly $\mathrm{N}$ in discriminating between osteitis condensans ilii and $\omega$ ankylosing spondylitis in doubtful cases. An experienced radiographer can produce satisfactory views. We had an interest at one time in sacro-iliac tomograms, but $\frac{C}{\mathscr{D}}$ our radiologist had reservations about the amount of $\mathbb{D}$ radiation to the pelvic area, especially as many of these? doubtful cases occur in women of child-bearing age.

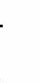
.

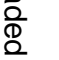

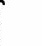

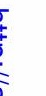

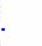

